\title{
INTEGRATED METHODOLOGY FOR SUPPLIER SELECTION: THE CASE OF A SPHYGMOMANOMETER MANUFACTURER IN TAIWAN
}

\author{
Z. H. $\mathrm{CHE}^{1}$, Y. F. $\mathrm{CHANG}^{2}$ \\ Department of Industrial Engineering and Management, \\ National Taipei University of Technology, Taipei, Taiwan

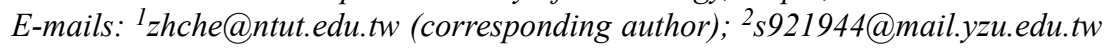

Received 13 May 2014; accepted 17 March 2015

\begin{abstract}
Supplier selection is a critical multi-criterion decision-making activity for successful supply chain management. This study involved developing an integrated supplier selection methodology, which is constructed using analytic network process, data envelopment analysis, and multiple objective particle swarm optimization. The proposed integrated methodology can account for multiple supplier selection criteria and set boundaries on weight value for multiple objective data envelopment analysis inputs and outputs. To solve the data envelopment analysis model, a new algorithm based on multiple objective particle swarm optimization is introduced, which embeds with tabu list and group mechanisms, and then, it is found to be superior to the compared algorithms in solving performance on three test functions and the illustrative case. In addition, the proposed integrated methodology was applied to a supplier selection problem of sphygmomanometer manufacturer in Taiwan to verify its applicability of decision-making process. The results show that the methodology can be implemented as an effective decision aid for supplier selection under multiple criteria with weight restrictions.
\end{abstract}

Keywords: supplier selection, decision-making, ANP, DEA, PSO, algorithm.

JEL Classification: C61, M11.

\section{Introduction}

Selecting appropriate suppliers is a critical challenge for enterprise purchasing managers in contemporary competitive conditions. Proper suppliers allow buyers to provide their customers with quality products and services at appropriate times and prices. Supplier selection plays a vital role in operational production and operation because most production costs stem from raw material purchases (Kokangul, Susuz 2009). Liu and Hai (2005), Hadi-Vencheh (2011), and Hadi-Vencheh and Niazi-Motlagh (2011) indicated that selecting suppliers is a difficult task for purchasing managers and Ustun and Demirtas (2008) said that supplier selection is a multi-criteria problem that may involve conflicting factors such as price and quality. To address such complicated decisionmaking tasks, a systematic methodology is needed to help decision makers scientifically select an alternative. 
Data envelopment analysis (DEA) is a mathematical programming model to evaluate the relative efficiencies by multiple inputs and outputs of decision making units (DMUs) and has been one of the most effective methods to the alternative assessment (Cooper et al. 2007). DEA has been widely and successfully applied in various industries (Cooper et al. 2007; Fried et al. 2008). It estimates the ratio of weighted outputs to weighted inputs to be the relative efficiency of each DMU, and then, comparing the efficiency of all DMUs (Lau 2013). In addition, Li and Reeves (1999) proposed a multiple objective DEA model that can be used to improve the discriminating power of traditional DEA methods. The supplier selection can be treated as an evaluation process with multiple inputs and outputs and a multiple objective DEA-based supplier selection model has been proposed in Che et al. (2011). DEA aims at finding the most favorable input and output weights for maximizing the efficiency of each DMU. From the practical decisionmaking perspective, however, the weight of each criterion in the DEA model should be restricted in the reasonable boundary to yield realistic efficiency scores. Moreover, distinct criteria are often mutually dependent in practical cases and can affect the outcome of the multi-criteria problem evaluation process. Inspire by this, this study continues and extends the work of Che et al. (2011) and introduces the analytic network process (ANP) approach in DEA model to determine the weight restriction for each input and output criterion based on decision-maker preferences with pondering interdependence interaction among these criteria to enhance the rationality of the model. Hence, the proposed DEA model can be used to find the appropriate suppliers whose weights of input and output criteria fit into the reasonable boundaries.

In addition, the proposed DEA model comprises multiple objectives that must be simultaneously achieved. In the past decade, numerous meta-heuristic algorithms have been proposed to solve multi-objective optimization problems and simultaneously optimize multiple factors such as the non-dominated sorting genetic algorithm (NDSGA) (Srinivas, Deb 1994), non-dominated sorting genetic algorithm II (NSGA-II; Deb et al. 2002), and non-dominated sorting particle swarm optimization (NSPSO) (Li 2003). Researchers have begun to focus on PSO to solve multi-objective problems and certain studies have proposed NSPSO as a method for such problems (Benabid et al. 2009; Liu 2009; Sedighizadeh et al. 2014); hence, we propose an NSPSO-based algorithm, namely NSPSOtg, which involves integrating tabu list and group mechanisms. The tabu list mechanism is employed to avoid scenarios in which all particles reach identical solutions and fall into the local solutions during evolution. The group mechanism is applied to prevent the located non-dominated solution sets from being over-concentrated, allowing the DEA model to be solved. Accordingly, this study involved developing a systematic supplier selection methodology, namely, hyADMOPSO, which integrates data envelopment analysis (DEA), analytic network processing (ANP), and multiple objective particle swarm optimization (MOPSO) to enhance enterprise competitiveness by facilitating the selection of appropriate suppliers. The characteristics of the methodology are the consideration of the multiple criteria and boundary constraints inherent to the decision making process, and the quality solving performance in the decision making environment with multiple objectives. Hence, the hyADMOPSO can be an effective methodology to be adopted by managers for supplier selection decision making. 
The remainder of the paper is organized as follows. The research background is explored in Section 1. Section 2 presents the construction of the hyADMOPSO methodology. The problem solving ability of NSPSOtg is evaluated in Section 3. In Section 4, the hyADMOPSO methodology is applied to supplier selection for a sphygmomanometer manufacturer, and the suitability of this methodology is discussed in Section 5. Conclusions are provided in the final section.

\section{Research background}

An effective supplier selection process is the key factor for organizational success (Wang, Che 2007; Hadi-Vencheh 2011; Ozkok, Tiryaki 2011). Supplier selection is a multi-criteria decision-making problem (Ustun, Demirtas 2008) in which organizations choose suppliers based on numerous concurrent criteria. Weber et al. (1991) investigated the citation frequency of each indicator in literature from 1967 to 1990, noting that the three vital criteria were cost, delivery, and quality. Ustun and Demirtas (2008) evaluated tangible and intangible factors before determining the optimal supply quantity. Liao and Rittscher (2007) used cost, quality, and time as the criteria for supplier evaluation. Wang and Che (2008) used cost and quality to address the problem of changed product parts when selecting suitable parts suppliers. Che (2012) clustered and selected suppliers based on production cost, product quality, and production time criteria. Che (2014) designed a methodology for the production and distribution planning, which integrates cost and time criteria.

DEA is an objective method for evaluating the relative efficiency of DMUs with identical multiple inputs and outputs by using production frontier. Various researchers have advanced alternate DEA models and numerous applications thereof. Certain pertinent studies of DEA method applications are considered herein as they relate to supplier selection, such as Ho et al. (2010), Chen (2011), and Falagario et al. (2012). To improve classical DEA model the criminating power, Li and Reeves (1999) proposed a multiobjective DEA model, minimizing the maximal quantity among all deviation variables and the deviation sum:

$$
\begin{aligned}
& \operatorname{Max} \theta_{k}=\sum_{r=1}^{R} u_{r} y_{r k}, \\
& \operatorname{Min} M, \\
& \operatorname{Min} \sum_{j=1}^{J} d_{j}, \\
& \text { s.t. } \sum_{i=1}^{I} v_{i} x_{i k}=1, \\
& \sum_{r=1}^{R} u_{r} y_{r j}-\sum_{i=1}^{I} v_{i} x_{i j}+d_{j}=0, \quad j=1, \ldots, J, \\
& u_{r} \geq 0, \quad r=1, \ldots, R, \\
& v_{i} \geq 0, \quad i=1, \ldots, I, \\
& d_{j} \geq 0 \text { and } M-d_{j} \geq 0, \quad j=1, \ldots, J,
\end{aligned}
$$


where $\theta_{k}$ is the relative efficiency value of the $k$ th DMU; $v_{i}$ and $u_{r}$ represent the weight values of input $i$ and output $r$, respectively; $x_{i k}$ and $y_{r k}$ represent the values of input $i$ and output $r$, respectively; $d_{j}$ represents the loss value of the $j$ th DMU; and $M$ represents the maximum loss value for all DMUs.

MOPSO involves using the Pareto front concept to describe the solution set and simultaneously address multiple objective problems. It is critical to select one of the numerous non-dominated solutions as the global optimum. Various scholars have used MOPSO to solve multiple objective optimization problems. These approaches suggest how to select the optimal local guide to facilitate updating the next flight velocity. Coello and Lechuga (2002) introduced a grid-based MOPSO algorithm, which is not necessarily ideal for the selected particle and can cause particle movement in the wrong direction during evolution. Parsopoulos and Vrahatis (2002) introduced the vector evaluated particle swarm optimization algorithm, which simplifies multiple objective functions into a single objective problem by assigning appropriate weight. Li (2003) developed the NSPSO model by adopting non-dominated sorting, crowded-comparison mechanism, and niche method. However, if the first search attains fewer non-dominated solutions compared with the total number of groups in NSPSO, certain solutions fairly distant from the Pareto front are retained in the next generation in addition to the close solutions. This affects the convergence of the algorithm. Therefore, we proposed the NSPSOtg algorithm, adding group and tabu list mechanisms to the basic NSPSO process.

\section{2. hyADMOPSO methodology}

The hyADMOPSO methodology is detailed in the following subsections.

\subsection{Identify supplier evaluation and selection criteria}

After referring to relevant literature, we summarized key reference criteria and distributed a questionnaire to industrial experts to determine the critical supplier selection criteria. The experts comprised professionals (e.g. procurement managers, CTOs) from relevant industries. The questionnaire employed a 5-point Likert scale; the degree of criterion importance was divided into "completely unimportant" =1, "somewhat unimportant" $=2$, "neither important nor unimportant" =3, "somewhat important" = 4, and "extremely important" $=5$. The criteria that attained high degrees of importance were preserved; those that did not be omitted.

\subsection{Determine the weight boundary of each criterion}

The questionnaire was released to experts to determine the relative importance between two criteria and calculate the weight boundary of each criterion according by using the ANP supermatrix approach. The ANP procedure is as following steps (Büyüközkan, Çifçi 2011; Che et al. 2012):

Step 1: Calculate the pairwise comparison matrix.

Each criterion is compared pairwise with respect to its effect on other criteria.

Step 2: Calculate the local priority vectors. 
The local priority vectors for each matrix are calculated using the eigenvector method: $A w=\lambda_{\max } w$. The $\lambda_{\max }$ is the largest eigenvalue of matrix $A$ and $w$ is the eigenvector.

Step 3: Test the consistency.

The $C R$ (consistency ratio) $=$ consistency index/random consistency index is used to evaluate the consistency of the weight assessment. If $C R \geq 0.1$, the decision makers must modify their judgments to generate a consistent comparison matrix.

Step 4: Construct the unweighted supermatrix.

All priority vectors from the matrices in the previous steps are arranged within an unweighted supermatrix.

Step 5: Derive the criteria weight boundaries.

The weighted supermatrix is calculated by multiplying the initial supermatrix by the cluster weight. Subsequently, the global weights are calculated $n$ times by multiplying the weighted supermatrix until the columns are adjusted. Regarding each specific criterion, the upper limit of the weight scope was set based on the maximal expert value and the lower limit was set based on the minimal expert value.

\subsection{Normalize data and develop the multiple objective DEA model}

For each input $x_{i}$ and output $y_{r}$ of each DMU, the normalization is by Formula (2):

$$
N\left(x_{i}\right)=x_{i} / \operatorname{Max}\left\{x_{i} \mid i=1, \ldots, I\right\} \text { and } N\left(y_{r}\right)=y_{r} / \operatorname{Max}\left\{y_{r} \mid r=1, \ldots, R\right\} .
$$

The ranking of each supplier is determined by the modified multiple objective DEA shown in Formula (3):

$$
\begin{aligned}
& f_{1}: \operatorname{Max} Z_{k}=\sum_{r=1}^{R} u_{r k} N\left(y_{r k}\right), \\
& f_{2}: \operatorname{Min} M, \\
& f_{3}: \operatorname{Min} \sum_{j=1}^{J} d_{j}, \\
& \text { s.t. } \sum_{r=1}^{R} u_{r k} N\left(y_{r j}\right)-\sum_{i=1}^{I} v_{i k} N\left(x_{i j}\right)+d_{j}=0, \quad j=1, \ldots, J, \\
& \sum_{i=1}^{I} v_{i k} N\left(x_{i k}\right)=1, \\
& U_{r} \geq u_{r k} \geq L_{r}, \quad r=1, \ldots, R, \\
& U_{i} \geq v_{i k} \geq L_{i}, \quad i=1, \ldots, I, \\
& M-d_{j} \geq 0, \quad j=1, \ldots, J, \\
& d_{j}>0, \quad j=1, \ldots, J,
\end{aligned}
$$

where $Z_{k}$ is the relative efficiency value of the $k$ th DMU; $v_{i k}$ and $u_{r k}$ represent the weight values of input $i$ and output $r$, respectively; $N\left(x_{i k}\right)$ and $N\left(y_{r k}\right)$ represent the normalized values of input $i$ and output $r$, respectively; $U_{i}$ and $L_{i}$ : represent the upper and lower limits of the weights of input $i ; U_{r}$ and $L_{r}$ : represent the upper and lower limits of the weights of output $r ; d_{j}$ represents the loss value of the $j$ th DMU; and $M$ represents the maximum loss value for all DMUs. 


\subsection{Develop the NSPSOtg for solving multiple objective DEA model}

\subsubsection{Code and create the initial population}

Regarding coding, each particle represents the weight values of input and output as shown in Figure 1. Then we randomly established an initial population, comprising $I$ particles and, the input and output weights were randomly generated; the scope of initial weight values were within the weight scope calculated using ANP.

\begin{tabular}{|c|c|c|c|c|c|}
\hline$v_{1}$ & $v_{2}$ & $v_{3}$ & $v_{4}$ & $\cdots$ & $v_{l}$ \\
\hline$u_{1}$ & $u_{2}$ & $u_{3}$ & $u_{4}$ & $\cdots$ & $u_{i}$ - input weight value \\
$u_{i}$ - output weight value
\end{tabular}

Fig. 1. Structure of particle

\subsubsection{Sort the non-dominated solution}

After calculating the objective values of all particles in the population by Formula (3), NSPSOtg compares the objective values of each solution with others to find a set of individuals that are the non-dominated solutions. The population will be screened and if the particles are not dominated by other particles, they will be moved out from the population and assigned as Level 1. In this way, the repeated screenings have brought the sequentially incremental value of level of whole particles in the population.

\subsubsection{Niche method}

The crowding distance is employed to be the niche method. When particle $i$ has a larger crowding distance, it yields more diversity among the solutions. Formula (4) shows the crowding distance calculation for particle $i$ (Deb et al. 2002), where the crowding distance between the two particles at the boundary is set as infinite:

$$
\operatorname{Crod}_{i}=\sum_{k}\left|f_{k i+1}-f_{k i-1}\right| /\left(\operatorname{Max}_{-} f_{k}-\operatorname{Min}_{-} f_{k}\right) \text {, }
$$

where Max $f_{k}$ and Min $f_{k}$ are the maximal and minimal values of particle $k$ on the frontline composed of non-dominated solutions set and $I$ is the total number of particles.

\subsubsection{Create the new population}

The new population creation process is based on the level and crowding distance of each particle. The individual with the lower level and lager crowding distance is preferred to select as a member of the new population. A new population with I particles will be created. Particles will be moved into the new population according to the ascending order of their levels until there are $I$ particles selected. When particles attain identical levels, they are judged based on the crowding distance, where large particles are preferred.

\subsubsection{Set a tabu list}

Another storage space is set at three times the size of $I$ particles to store the three most recent generations of the optimal values of each particle. This list is continually updated. Once the latest generation of optimal value is generated, the oldest generation the optimal value is removed. 


\subsubsection{Update the velocities and positions}

Formula (5) shows the velocity calculation method and position update (Clerc 1999). The solutions must be distinct from those on the tabu list after each particle update or the particle velocity and position updates must be repeated:

$$
\begin{aligned}
& v_{i d}^{j+1}=k\left(v_{i d}^{j}+\phi_{1} \times \operatorname{rand}() \times\left(p_{i d}-x_{i d}^{j}\right)+\phi_{2} \times \operatorname{rand}() \times\left(g_{d}-x_{i d}^{j}\right)\right), \\
& k=2 /\left(\left|2-\phi-\sqrt{\phi^{2}-4 \phi}\right|\right), \phi=\phi_{1}+\phi_{2}, \phi>4, \\
& x_{i d}^{j+1}=x_{i d}^{j}+v_{i d}^{j+1},
\end{aligned}
$$

where, $v_{i d}^{j}$ and $x_{i d}^{j}$ represent the velocity and position of the $j$ th generation of particle $i$ at dimension $d ; p_{i d}$ represents the position of the optimal value of particle $i ; g_{d}$ represents the position of the optimal value of the entire population; $k$ represents the constriction factor; $\phi_{1}$ and $\phi_{2}$ represent acceleration constants; and rand () represents the independent random variable distributed uniformly within $[0,1]$.

\subsubsection{Group mechanism}

The group mechanism generates a new population by combining a population derived after the position update with the original population. Non-dominated sorting is conducted in this new population and non-dominated Level 1 solutions are temporarily stored in an external storage space. We identified non-dominated solutions among these temporarily stored solutions and those originally stored in the external space, retaining the non-dominated solutions and removing the dominated solutions. The scope of group with radius $r$ was assigned to each particle. One of the solutions is selected as the center of the groups and any other solution falling within this scope of the group is removed. The selection of the group center and the search for the scope of group is conducted for all remaining solutions until all solutions are identified. Only solutions labeled as the group centers are preserved to ensure the diversity of the determined non-dominated solutions. Formula (6) shows the group radius $r$ :

$$
r=\operatorname{Min}\left\{\left(\operatorname{Max}_{-} f_{k}-\operatorname{Min}_{-} f_{k}\right) \mid k \in 1, \ldots, K\right\} / 2 N,
$$

where Max $\_f_{k}$ and Min $f_{k}$ represent the maximal and minimal values of each particle at objective $k$ on the frontline composed of the non-dominated solutions set and $N$ represents the number of particles.

\subsubsection{Derive the population of final solutions}

When the number of evolutions doesn't reach the preset number of generations, the evolution continues; otherwise, the non-dominated solution set retained in the external storage space is the set of optimal solutions.

\section{Evaluation of problem-solving performance of NSPSOtg}

In this section, the problem-solving performance levels of the proposed NSPSOtg algorithm was verified using three standard test functions and compared using two common algorithms: NSGA-II and NSPSO. 


\subsection{Test function and performance indicator}

Table 1. Test functions

\begin{tabular}{|c|c|c|c|}
\hline Problem & $\begin{array}{l}\text { Range of } \\
\text { variable } x\end{array}$ & Objective function (minimize) & Reference \\
\hline $\mathrm{SCH}$ & {$\left[-10^{3}, 10^{3}\right]$} & $\begin{array}{l}f_{1}(x)=x^{2} \\
f_{2}(x)=(x-2)^{2}\end{array}$ & $\begin{array}{l}\text { (Tsai et al. 2010; Gong et al. } \\
\text { 2010; Rodríguez et al. 2009) }\end{array}$ \\
\hline KUR & {$[-5.5]$} & $\begin{array}{l}f_{1}(x)=\sum_{i=1}^{n-1}\left(-10 \exp \left(-0.2 \sqrt{x_{i}^{2}+x_{i+1}^{2}}\right)\right) \\
f_{2}(x)=\sum_{i=1}^{n}\left(\left|x_{i}\right|^{0.8}+5 \sin x_{i}^{3}\right)\end{array}$ & $\begin{array}{l}\text { (Gong et al. 2010; Wang } \\
\text { et al. 2009; Salazar-Lechuga, } \\
\text { Rowe 2005) }\end{array}$ \\
\hline ZDT2 & {$[0.1]$} & $\begin{array}{l}f_{1}(x)=x_{1} \\
f_{2}(x)=g(x)\left[1-\left(x_{1} / g(x)\right)^{2}\right] \\
g(x)=1+9\left(\sum_{i=2}^{n} x_{i}\right) /(n-1)\end{array}$ & $\begin{array}{l}\text { (Tsai et al. 2010; Gong et al. } \\
\text { 2010; Tripathi et al. 2007) }\end{array}$ \\
\hline
\end{tabular}

Table 1 lists descriptions of three test functions: SCH (Schaffer 1985), KUR (Kursawe 1991), and ZDT2 (Zitzler et al. 2000). Zitzler et al. (2000) introduced three major evaluation targets as performance level indicators for algorithms:

(a) Accuracy: The final non-dominated solution set should comprise numerous solutions converged to the Pareto front, that includes Number of non-dominated solutions (NNS) (Rahimi-Vahed et al. 2007), Number of Pareto solutions (NPS) (Schaffer 1985), and Error ratio (ER) (Rahimi-Vahed et al. 2007) indicators. Formula (7) shows the ER:

$$
E R=\sum_{i=1}^{n} e_{i} / n
$$

where $n$ is the number of non-dominated solutions; $e_{i}$ is a binary variable, if nondominated solution $i$ is a Pareto solution, and $e_{i}=0$; otherwise, $e_{i}=1$. An $E R$ closes to 1 indicates that few non-dominated solutions converged to the Pareto front.

(b) Distribution: The Diversity metric $(D M)$ is used to evaluate the diversity among non-dominated solutions and as follows (Deb et al. 2002):

$$
D M=\left(d_{f}+d_{l}+\sum_{i=1}^{n-1}\left|d_{i}-\bar{d}\right|\right) /\left(d_{f}+d_{l}+(n-1) \bar{d}\right),
$$

where $n$ is the number of non-dominated solutions determined using the algorithm; $d_{f}$ and $d_{l}$ are the distances between the solutions at both ends of the Pareto front and their neighboring non-dominated solutions; $d_{i}$ is the distance between a non-dominated solution and its neighboring solution; and $\frac{d}{d}$ is the average value of $d_{i}$. 
(c) Spread: The Maximum Spread (MS) is used to measure the spread of the nondominated solution set and as follows (Zitzler et al. 2000; Rahimi-Vahed et al. 2007):

$$
M S=\sqrt{\sum_{m=1}^{M}\left(\max _{i=1}^{n} f_{m}^{i}-\min _{i=1}^{n} f_{m}^{i}\right)^{2}},
$$

where $n$ is the number of non-dominated solutions and $M$ is the number of objective functions. The larger the $M S$ is, the more optimal the spread of the determined nondominated solution is.

\subsection{Setting the parameters}

We developed the NSPSOtg model by using parameters such as $N$ (number of particles), $G$ (number of generations), $\phi_{1}$ and $\phi_{2}$ (acceleration constants), and $V_{\max }$ (maximal velocity). Li (2003) set the $N$ and $G$ of an NSPSO model as 200 and 100, respectively, to compare it with the NSGA-II. Rahimi-Vahed et al. (2007) set $N$ and $G$ of the multiple objective genetic algorithm as 50 and 50 to solve the problem of the sequence of a small-scale assembly line. Tripathi et al. (2007) set the $N$ and $G$ of NSPSO and NSGAII as 100 and 250, respectively. Clerc (1999) and Clerc and Kennedy (2002) suggested that the sum of $\phi_{1}$ and $\phi_{2}$ should be larger than four to ensure the optimization of the particle swarm acceleration function by adding a constriction factor. However, none of these studies specified optimal parameter combination; the only suggestion was that the two parameters be set as $\phi_{1}=\phi_{2}=2.05$. Zhang et al. (2005) proposed that when $\phi_{1}=2.8$ and $\phi_{2}=1.3$, the convergence of the population can be accelerated. Clerc and Kennedy (2002) suggested that strong good convergence ability can be attained without restricting the $V_{\max }$. However, Eberhart and Shi (2000) argued that the execution would improve if velocity limitation was considered for particle swarm optimization and combined with the constriction factor. We integrated the aforementioned scholar experiences and Table 2 shows the factors and levels used in the experimental design. Each parameter combination was repeated 10 times to obtain the mean $E R$ value for comparative analysis. Table 3 lists the results of the experimental design and shows that when the NSPSOtg parameter combination $G, N, \phi_{1}, \phi_{2}$, and $V_{\max }$ is set at 250, 200, $2.05,2.05$, and \pm 1 , respectively, it eventually yields an accurate non-dominated solution set. Therefore, these parameter settings were employed to comparatively analyze the performance levels of various algorithms.

Table 2. Experimental design for factors

\begin{tabular}{cccc}
\hline Factor & Level 1 & Level 2 & Level 3 \\
\hline$G$ & 50 & 100 & 250 \\
\hline$N$ & 50 & 100 & 200 \\
\hline$\phi_{1}, \phi_{2}$ & $(2.05,2.05)$ & $(2.8,1.3)$ & - \\
\hline$V_{\max }$ & no limit & \pm 1 & - \\
\hline
\end{tabular}


Z. H. Che, Y. F. Chang. Integrated methodology for supplier selection ...

Table 3. Experimental design for NSPSOtg's parameters

\begin{tabular}{ccccccccccc}
\hline & & \multicolumn{3}{c}{$G 50$} & \multicolumn{3}{c}{100} & \multicolumn{3}{c}{250} \\
\hline$\left(\phi_{1}, \phi_{2}\right) V_{\max }$ & $N 50$ & 100 & 200 & 50 & 100 & 200 & 50 & 100 & 200 \\
\hline \multirow{2}{*}{$(2.05,2.05)$} & no limit & 0.307 & 0.348 & 0.335 & 0.397 & 0.347 & 0.301 & 0.347 & 0.319 & 0.282 \\
\cline { 2 - 11 } & \pm 1 & 0.416 & 0.381 & 0.283 & 0.381 & 0.413 & 0.297 & 0.388 & 0.317 & 0.231 \\
\hline \multirow{2}{*}{$(2.8,1.3)$} & no limit & 0.298 & 0.311 & 0.343 & 0.459 & 0.357 & 0.355 & 0.329 & 0.339 & 0.301 \\
\cline { 2 - 10 } & \pm 1 & 0.385 & 0.372 & 0.364 & 0.429 & 0.346 & 0.349 & 0.440 & 0.347 & 0.309 \\
\hline
\end{tabular}

\subsection{Analyzing the results}

Table 4. Comparisons of solving performances among different algorithms on test functions

\begin{tabular}{cccccccl}
\hline \multicolumn{2}{l}{ Indicator } & NSPSOtg & NSPSO & NSGA-II & F-value & p-value & Ranking \\
\hline SCH & NNS & 197.867 & 197.467 & 82.333 & 2170.810 & $4.07 \mathrm{E}-43$ & NSPSOtg $=$ NSPSO $>$ NSGA-II \\
\hline & NPS & 197.733 & 197.467 & 81.533 & 2110.579 & $7.31 \mathrm{E}-43$ & NSPSOtg $=$ NSPSO $>$ NSGA-II \\
\hline & ER & 0.001 & 0.000 & 0.009 & 11.335 & $1.16 \mathrm{E}-04$ & NSPSO $=$ NSPSOtg $<$ NSGA-II \\
\hline & DM & 0.948 & 1.071 & 0.978 & 29.493 & $9.97 \mathrm{E}-09$ & NSPSOtg $<$ NSGA-II $<$ NSPSO \\
\hline KUR & NNS & 5.465 & 1.031 & 5.628 & 582.145 & $2.39 \mathrm{E}-31$ & NSGA-II $>$ NSPSOtg $>$ NSPSO \\
\hline & NPS & 61.800 & 19.667 & 36.933 & 21.189 & $4.34 \mathrm{E}-07$ & NSPSOtg $>$ NSGA-II $>$ NSPSO \\
\hline & ER & 0.237 & 0.333 & 0.136 & 3.929 & 0.027 & NSGA-II $<$ NSPSOtg $=$ NSPSO \\
\hline DM & 0.911 & 0.972 & 0.918 & 16.479 & $5.21 \mathrm{E}-06$ & NSPSOtg $=$ NSGA-II $<$ NSPSO \\
\hline ZD & 12.783 & 4.071 & 8.922 & 99.533 & $1.16 \mathrm{E}-16$ & NSPSOtg $>$ NSGA-II $>$ NSPSO \\
\hline NNS & 159.267 & 49.267 & 29.200 & 54.952 & $1.88 \mathrm{E}-12$ & NSPSOtg $>$ NSPSO $>$ NSGA-II \\
\hline & NPS & 151.267 & 21.267 & 1.333 & 72.603 & $2.34 \mathrm{E}-14$ & NSPSOtg $>$ NSPSO $>$ NSGA-II \\
\hline ER & 0.065 & 0.582 & 0.954 & 53.409 & $6.78 \mathrm{E}-12$ & NSPSOtg $<$ NSPSO $<$ NSGA-II \\
\hline DM & 0.816 & 0.918 & 0.949 & 34.121 & $1.58 \mathrm{E}-09$ & NSPSOtg $<$ NSPSO $<$ NSGA-II \\
\hline MS & 1.329 & 0.898 & 1.321 & 28.278 & $1.66 \mathrm{E}-08$ & NSPSOtg $=$ NSGA-II $>$ NSPSO \\
\hline
\end{tabular}

Table 4 shows the comparison of problem-solving performance levels among NSPSOtg and NSGA-II, and NSPSO (number of experiments $n=30$ and $\alpha=0.05$ ). First, regarding the SCH problem, the NNS and NPS indicators show that the NNS obtained using NSPSOtg and NSPSO was significantly superior compared with that of NSGA-II. The $E R$ indicators of the algorithms were all close to 0, suggesting that the non-dominated $\mathrm{SCH}$ problem solutions obtained by these algorithms accurately converge to the Pareto front. In terms of distribution, the $D M$ showed that the NSPSOtg was slightly superior compared with NSGA-II and NSPSO demonstrated poor diversity $(D M>1)$. The $M S$ indicator showed that NSPSOtg and NSGA-II demonstrated the strongest spread. Analogously, on average, the performanc indicators showed that NSPSOtg is superior to NSPSO and NSGA-II in terms of accuracy, distribution, and spread on the KUR and ZDT2 problems. 


\section{Implementation of hyADMOPSO methodology in supplier selection}

To further verify the suitability of the proposed hyADMOPSO methodology, we empirically investigated a case, where, Company $\mathrm{A}$ is a sphygmomanometer manufacturer that uses 16 part suppliers.

\subsection{Determination of the supplier selection criteria and weight of each criterion}

The key reference criteria are summarized by referring to relevant literature, that affect supplier evaluation and selection. Six experts are selected through the group discussion of managers of the case company. The members of selected experts include three internal managers of case company and three external experts from relevant industries. They scored the criteria by questionnaires and four major dimensions and eight assessment criteria are determined. If the applicability of the data from the expert is doubtful, he/she will be asked to provide an explanation and/or fix the judgment. The relative weights and weighted boundaries of the selected indicators, then, is determined via the ANP model (Table 5).

Table 5. Supplier selection criteria and weight scope of each criterion

\begin{tabular}{llcccc}
\hline \multirow{2}{*}{ Criterion } & \multicolumn{1}{c}{ Sub-criterion } & Input/Output & Average & Max & Min \\
\hline \multirow{2}{*}{ Cost } & Purchasing cost (Pc) & Input 1 & 0.108 & 0.314 & 0.032 \\
\cline { 2 - 6 } & Transportation cost (Tc) & Input 2 & 0.068 & 0.245 & 0.010 \\
\hline Quality & Defective rate (Qdr) & Input 3 & 0.145 & 0.256 & 0.047 \\
\hline Service & Maintenance turnaround time (Mtt) & Input 4 & 0.062 & 0.128 & 0.008 \\
\hline Time & On-time delivery rate (Odr) & Output 1 & 0.241 & 0.369 & 0.096 \\
\hline Quality & Reliability (Re) & Output 2 & 0.235 & 0.257 & 0.037 \\
\hline Service & Supply capacity (Sc) & Output 3 & 0.077 & 0.235 & 0.017 \\
\cline { 2 - 6 } & Warranty time (Wt) & Output 4 & 0.064 & 0.132 & 0.008 \\
\hline
\end{tabular}

\subsection{Data normalization and results of supplier selection by NSPSOtg}

Table 6 lists the input and output data that was normalized and introduced in the multiple objective DEA models. Through problem solving with NSPSOtg, the results are shown in Table 7. For Suppliers 3, 9 and 16 come up with non-dominated solutions of Level 1, these three suppliers are the most preferred choices. Supplier 7 has the worst ranking because of its Level 5 non-dominated solutions. 
Table 6. Data of input and output items for suppliers

\begin{tabular}{ccccccccc}
\hline Supplier & $\begin{array}{c}\text { Pc } \\
\text { (dollars) }\end{array}$ & $\begin{array}{c}\text { Tc } \\
\text { (dollars) }\end{array}$ & $\begin{array}{c}\text { Qdr } \\
(\%)\end{array}$ & $\begin{array}{c}\text { Mtt } \\
\text { (days) }\end{array}$ & $\begin{array}{c}\text { Re } \\
(\%)\end{array}$ & $\begin{array}{c}\text { Sc } \\
\text { (units) }\end{array}$ & $\begin{array}{c}\text { Wt } \\
\text { (months) }\end{array}$ & $\begin{array}{c}\text { Odr } \\
(\%)\end{array}$ \\
\hline 1 & 20 & 4 & 2 & 7 & 75 & 900 & 6 & 80 \\
\hline 2 & 10 & 1 & 1 & 7 & 60 & 800 & 6 & 80 \\
\hline 3 & 15 & 2 & 1.5 & 7 & 70 & 1000 & 18 & 90 \\
\hline 4 & 20 & 4 & 2 & 14 & 90 & 900 & 12 & 90 \\
\hline 5 & 15 & 2 & 2.5 & 7 & 70 & 800 & 6 & 80 \\
\hline 6 & 11 & 1 & 1 & 14 & 65 & 800 & 6 & 90 \\
\hline 7 & 11 & 1 & 1.5 & 7 & 60 & 800 & 6 & 80 \\
\hline 8 & 12 & 2 & 1.5 & 14 & 65 & 800 & 6 & 85 \\
\hline 9 & 15 & 4 & 1.5 & 14 & 90 & 1000 & 12 & 90 \\
\hline 10 & 10 & 1 & 1.5 & 7 & 60 & 900 & 12 & 85 \\
\hline 11 & 10 & 2 & 1 & 7 & 70 & 800 & 6 & 88 \\
\hline 12 & 15 & 2 & 1.5 & 14 & 70 & 800 & 6 & 85 \\
\hline 13 & 12 & 3 & 2 & 7 & 85 & 1000 & 12 & 99 \\
\hline 14 & 20 & 4 & 2.5 & 14 & 80 & 1000 & 12 & 90 \\
\hline 15 & 10 & 1 & 1 & 14 & 65 & 800 & 6 & 85 \\
\hline 16 & 20 & 5 & 2.5 & 14 & 95 & 1000 & 18 & 90 \\
\hline & & & & & & & & \\
\hline
\end{tabular}

Table 7. Results of sorting suppliers by multiple objective DEA

\begin{tabular}{cccccccccccc}
\hline Supplier & $f_{1}$ & $f_{2}$ & $f_{3}$ & Level & Ranking & Supplier & $f_{1}$ & $f_{2}$ & $f_{3}$ & Level Ranking \\
\hline 1 & 0.603 & 0.572 & 4.151 & 3 & 8 & 9 & 0.855 & 0.618 & 3.944 & 1 & 1 \\
\hline 2 & 0.995 & 0.999 & 6.539 & 4 & 13 & 10 & 0.995 & 0.783 & 5.366 & 2 & 4 \\
\hline 3 & 0.999 & 0.652 & 4.452 & 1 & 1 & 11 & 0.995 & 0.914 & 5.843 & 3 & 8 \\
\hline 4 & 0.639 & 0.513 & 3.372 & 2 & 4 & 12 & 0.689 & 0.724 & 4.663 & 3 & 8 \\
\hline 5 & 0.716 & 0.721 & 4.794 & 4 & 13 & 13 & 0.982 & 0.690 & 4.662 & 2 & 4 \\
\hline 6 & 0.952 & 0.932 & 5.781 & 3 & 8 & 14 & 0.568 & 0.432 & 3.003 & 2 & 4 \\
\hline 7 & 0.886 & 1.067 & 6.881 & 5 & 16 & 15 & 0.982 & 0.954 & 5.912 & 4 & 13 \\
\hline 8 & 0.743 & 0.719 & 4.778 & 3 & 8 & 16 & 0.665 & 0.426 & 2.931 & 1 & 1 \\
\hline
\end{tabular}

\section{Discussion}

Table 8. Comparisons of problem-solving performances among different algorithms on the case

\begin{tabular}{ccccccc}
\hline \multirow{2}{*}{$\begin{array}{l}\text { Performance } \\
\text { indicator }\end{array}$} & NSPSOtg & NSPSO & NSGA- & \multicolumn{2}{c}{ ANOVA } & Ranking \\
\cline { 5 - 6 } & & & II & F-value & p-value & \\
\hline NNS & 26.758 & 5.200 & 11.092 & 1354.592 & 0.000 & NSPSOtg $>$ NSGA-II $>$ NSPSO \\
\hline NPS & 26.058 & 0.000 & 1.767 & 4596.573 & $6.52 \mathrm{E}-50$ & NSPSOtg $>$ NSGA-II $>$ NSPSO \\
\hline ER & 0.115 & 1.000 & 0.885 & 3572.505 & $1.26 \mathrm{E}-47$ & NSPSOtg $<$ NSGA-II $<$ NSPSO \\
\hline DM & 0.857 & 0.909 & 0.872 & 49.309 & $9.53 \mathrm{E}-12$ & NSPSOtg $<$ NSGA-II $<$ NSPSO \\
\hline MS & 1.105 & 0.735 & 0.585 & 105.531 & $4.17 \mathrm{E}-17$ & NSPSOtg $>$ NSPSO $>$ NSGA-II \\
\hline
\end{tabular}


Table 8 lists the comparisons among NSPSOtg, NSGA-II, and NSPSO. In terms of accuracy, NSPSOtg yields more non-dominated solutions and superior NNS, NPS and $E R$ values compared with the other algorithms. In terms of distribution, NSPSOtg was slightly superior to others in $D M$. The $M S$ indicator showed that NSPSOtg had the strongest spread. These performance indicators suggest that NSPSOtg is superior to NSPSO and NSGA-II in terms of accuracy, distribution, and spread in the proposed problem; therefore we summarized the non-dominated solution set of suppliers found using NSPSOtg in a multiple objective DEA model and performed non-dominated solution sorting to determine which DMUs are efficient.

Table 9. Results of sorting suppliers by classical DEA

\begin{tabular}{cccccccccc}
\hline Supplier & $f_{1}$ & $f_{2}$ & $f_{3}$ & Ranking & Supplier & $f_{1}$ & $f_{2}$ & $f_{3}$ & Ranking \\
\hline 1 & 0.899 & 1.199 & 9.899 & 11 & 9 & 0.976 & 1.001 & 5.151 & 9 \\
\hline 2 & 1.000 & 1.238 & 7.482 & 1 & 10 & 1.000 & 1.249 & 8.467 & 1 \\
\hline 3 & 1.000 & 1.001 & 6.739 & 1 & 11 & 1.000 & 1.088 & 6.539 & 1 \\
\hline 4 & 0.732 & 0.474 & 2.471 & 14 & 12 & 0.718 & 0.616 & 3.668 & 15 \\
\hline 5 & 0.965 & 0.897 & 6.321 & 10 & 13 & 1.000 & 0.975 & 7.887 & 1 \\
\hline 6 & 1.000 & 1.517 & 8.369 & 1 & 14 & 0.633 & 0.599 & 4.949 & 16 \\
\hline 7 & 0.995 & 3.167 & 18.263 & 8 & 15 & 1.000 & 1.413 & 7.601 & 1 \\
\hline 8 & 0.814 & 0.919 & 5.589 & 13 & 16 & 0.825 & 0.442 & 2.306 & 12 \\
\hline
\end{tabular}

Table 10. Weights of suppliers by multiple objective DEA and classical DEA

\begin{tabular}{lllllllll}
\hline \multicolumn{7}{c}{ Multiple objective DEA } \\
\hline Supplier & v1 & v2 & v3 & v4 & u1 & u2 & u3 & u4 \\
\hline 1 & 0.1515 & 0.0100 & 0.1399 & 0.1236 & 0.0370 & 0.0926 & 0.0321 & 0.0960 \\
\hline 2 & 0.2943 & 0.1368 & 0.2469 & 0.1199 & 0.1898 & 0.1252 & 0.0924 & 0.0999 \\
\hline 3 & 0.2254 & 0.0155 & 0.2096 & 0.0644 & 0.0546 & 0.0512 & 0.1320 & 0.1203 \\
\hline 4 & 0.1293 & 0.0100 & 0.2158 & 0.0233 & 0.0428 & 0.0170 & 0.1046 & 0.0960 \\
\hline 5 & 0.2082 & 0.1659 & 0.0470 & 0.1275 & 0.1900 & 0.0184 & 0.0088 & 0.1003 \\
\hline 6 & 0.2733 & 0.1787 & 0.2505 & 0.0469 & 0.2540 & 0.0170 & 0.1279 & 0.0960 \\
\hline 7 & 0.2916 & 0.2420 & 0.1126 & 0.1138 & 0.1623 & 0.0472 & 0.0203 & 0.1839 \\
\hline 8 & 0.2875 & 0.0447 & 0.2024 & 0.0214 & 0.0370 & 0.1516 & 0.0565 & 0.0960 \\
\hline 10 & 0.2379 & 0.0138 & 0.2006 & 0.0234 & 0.1021 & 0.0170 & 0.1259 & 0.0960 \\
\hline 11 & 0.3139 & 0.0100 & 0.2134 & 0.0925 & 0.0906 & 0.1073 & 0.1266 & 0.1089 \\
\hline 12 & 0.3023 & 0.1282 & 0.2253 & 0.0814 & 0.2362 & 0.0205 & 0.1320 & 0.1098 \\
\hline 13 & 0.2206 & 0.1554 & 0.1277 & 0.0290 & 0.1770 & 0.0170 & 0.0108 & 0.0960 \\
\hline 14 & 0.3136 & 0.0611 & 0.068 & 0.1074 & 0.1937 & 0.0170 & 0.0616 & 0.0960 \\
\hline 15 & 0.1592 & 0.0100 & 0.1115 & 0.0545 & 0.0370 & 0.0174 & 0.0800 & 0.0960 \\
\hline 16 & 0.3109 & 0.1589 & 0.2560 & 0.0436 & 0.2315 & 0.0289 & 0.1320 & 0.1187 \\
\hline & 0.1592 & 0.0100 & 0.1115 & 0.0525 & 0.0370 & 0.0174 & 0.0800 & 0.0960 \\
\hline
\end{tabular}


End of Table 10

\begin{tabular}{lllllllll}
\hline \multicolumn{7}{c}{ Classical DEA } \\
\hline 1 & 0.0000 & 0.0000 & 0.0000 & 1.9999 & 0.0000 & 0.9999 & 0.0000 & 0.0000 \\
\hline 2 & 1.4249 & 0.4999 & 0.3125 & 0.1249 & 0.0000 & 0.0000 & 0.0000 & 1.2374 \\
\hline 3 & 1.3333 & 0.0000 & 0.0000 & 0.0000 & 0.0000 & 0.0000 & 0.9999 & 0.0000 \\
\hline 4 & 0.7141 & 0.0000 & 0.3572 & 0.0000 & 0.5089 & 0.0000 & 0.3749 & 0.0000 \\
\hline 5 & 0.0000 & 0.8618 & 0.0000 & 1.3104 & 1.3103 & 0.0000 & 0.0000 & 0.0000 \\
\hline 6 & 0.0000 & 0.0000 & 2.4147 & 0.0340 & 0.0000 & 0.0000 & 0.6988 & 0.8437 \\
\hline 7 & 0.0000 & 1.0413 & 0.0000 & 1.5834 & 1.5832 & 0.0000 & 0.0000 & 0.0000 \\
\hline 8 & 1.5708 & 0.1436 & 0.0000 & 0.0000 & 0.0000 & 0.0000 & 0.0000 & 0.9482 \\
\hline 10 & 0.0000 & 0.0000 & 1.6666 & 0.0000 & 0.6785 & 0.0000 & 0.4999 & 0.0000 \\
\hline 11 & 0.0000 & 1.2499 & 1.2499 & 0.0000 & 0.0000 & 0.7142 & 0.5357 & 0.0000 \\
\hline 12 & 1.9999 & 0.0000 & 0.0000 & 0.0000 & 0.0000 & 0.0000 & 0.0989 & 1.0878 \\
\hline 13 & 0.0000 & 0.5125 & 1.1541 & 0.1025 & 0.9743 & 0.0000 & 0.0000 & 0.0000 \\
\hline 14 & 0.2500 & 0.1874 & 0.0000 & 1.4749 & 0.0000 & 0.9999 & 0.0000 & 0.0000 \\
\hline 15 & 0.6665 & 0.0000 & 0.3334 & 0.0000 & 0.4749 & 0.0000 & 0.3499 & 0.0000 \\
\hline 16 & 0.0000 & 0.2939 & 2.3529 & 0.0000 & 1.1176 & 0.0000 & 0.7058 & 0.0000 \\
\hline
\end{tabular}

In addition, we compared the results (Table 7) with those attained using the traditional DEA (Table 9). The multiple objective DEA model maximizes its DMU efficiency and accounts for other DMUs during weight calculation such that the total variance and maximal variance are minimized. Thus, the results show that the efficient DMU obtained using the traditional DEA is not necessarily the ideal DMU for use in the multiple objective DEA model. For example, Supplier 13 is ranked first in the traditional

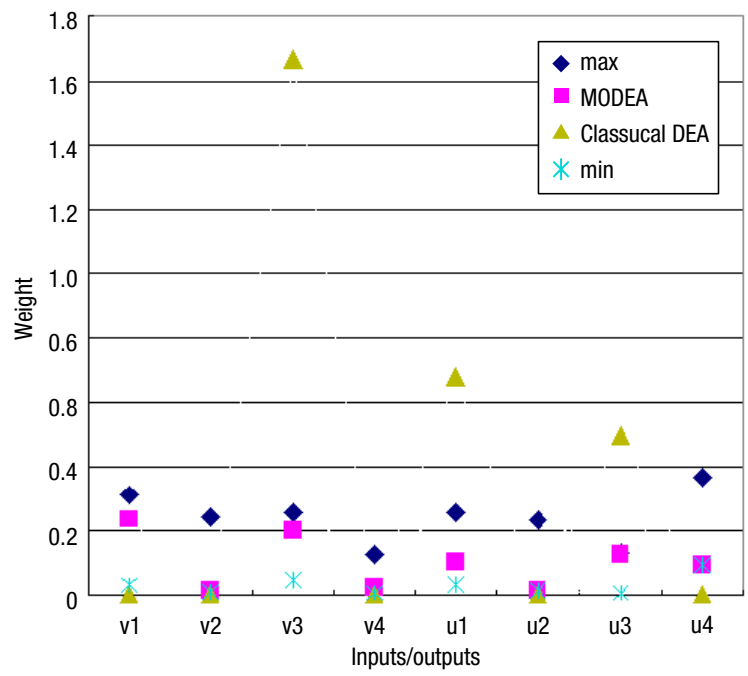

Fig. 2. Weight boundaries of inputs and outputs for Supplier 9 
DEA model but fourth in the multiple objective DEA model. Moreover, comparing $f_{2}$ and $f_{3}$ for all DMUs showed that the weights calculated using multiple objective DEA models typically yield a decreased total variance and maximal variance. The input and output weights were obtained by multiple objective DEA models relatively benefit all DMUs, such that the disadvantages of the traditional DEA are focused only on favorable evaluation conditions. Table 10 lists the weights results for all suppliers, and Figure 2 shows the weight boundaries of the inputs and outputs for Supplier 9. Because the multiple objective DEA model was developed based on the scope of weight suggested by industrial experts with ANP approach, reasonable input and output weights can be generated that address the question scenario.

\section{Conclusions}

Supplier evaluation and selection is a critical process for integrating the expertise and experiences of business managers and their supply chain partners, allowing enterprises to reduce costs and generate profits. Thus, supplier evaluation and selection is vital to business competition. We developed a supplier evaluation and selection methodology that integrated ANP, multiple objective DEA, and MOPSO, namely, hyADMOPSO. By integrating ANP and multiple objective DEA, all influential factors were considered and the scope of weight was concluded based on the relative weights suggested in the expert comments. In addition to maximizing the efficiency of its own DMU, multiple objective DEA accounts for other DMUs to ensure that the total and maximal variances are minimized. Furthermore, to effectively solve multiple objective DEA problems, an NSPSO-based MOPSO algorithm, namely, NSPSOtg was introduced. By using three test functions, we verified that NSPSOtg demonstrates satisfactory problem-solving performance levels. Finally, the hyADMOPSO methodology was applied to select suppliers for a sphygmomanometer manufacturer; the results show that the methodology is applicable to select appropriate suppliers when multiple criteria are presented.

Throughout the proposed methodology, although it used for the supplier selection decision in this paper, it can be applied for making the quality decision in other fields such as location selection, project evaluation, and evaluation of energy system. However, in its current form, it may not be introduced into to solving the more complex supplier selection problems. For instance, the hyADMOPSO can not gain the quality solution in the supplier selection problems when decision makers face with uncertain data, capacity restriction, and quality discount situations. In the future studies, the proposed methodology will be extended to these complex situations.

\section{References}

Benabid, R.; Boudour, M.; Abido, M. A. 2009. Optimal location and setting of SVC and TCSC devices using non-dominated sorting particle swarm optimization, Electric Power Systems Research 79(12): 1668-1677. http://dx.doi.org/10.1016/j.epsr.2009.07.004

Büyüközkan, G.; Çifçi, G. 2011. A novel fuzzy multi-criteria decision framework for sustainable supplier selection with incomplete information, Computers in Industry 62(2): 164-174.

http://dx.doi.org/10.1016/j.compind.2010.10.009 
Che, Z. H. 2012. Clustering and selecting suppliers based on simulated annealing algorithms, Computers and Mathematics with Applications 63(1): 228-238.

http://dx.doi.org/10.1016/j.camwa.2011.11.014

Che, Z. H.; Chiang, T. A.; Che, Z. G. 2012. Using analytic network process and turbo particle swarm optimization algorithm for non-balanced supply chain planning considering supplier relationship management, Transactions of the Institute of Measurement and Control 34(6): 720-735. http://dx.doi.org/10.1177/0142331211402901

Che, Z. H. 2014. A particle swarm optimization algorithm for solving unbalanced supply chain planning problems, Applied Soft Computing 12(4): 1279-1287.

http://dx.doi.org/10.1016/j.asoc.2011.12.006

Che, Z. H.; Chiang, T. A.; Wang, H. S.; Chang, Y. F. 2011. Development and application of an integrated multi-objective methodology for supplier selection, International Journal of the Physical Sciences 6(25): 5951-5960. http://dx.doi.org/10.5897/IJPS11.732

Chen, Y. J. 2011. Structured methodology for supplier selection and evaluation in a supply chain, Information Sciences 181(9): 1651-1670. http://dx.doi.org/10.1016/j.ins.2010.07.026

Clerc, M. 1999. The swarm and the queen: towards a deterministic and adaptive particle swarm optimization, in IEEE Congress on Evolutionary Computation, 6-9 July 1999, Washington, DC, USA, 1951-1957. http://dx.doi.org/0.1109/CEC.1999.785513

Clerc, M.; Kennedy, J. 2002. The particle swarm - explosion, stability, and convergence in a multidimensional complex space, IEEE Transactions on Evolutionary Computation 6(1): 58-73. http://dx.doi.org/10.1109/4235.985692

Coello, C. A.; Lechuga, M. S. 2002. MOPSO: a proposal for multiple objective particle swarm optimization, in IEEE Congress on Evolutionary Computation, 12-17 May 2002, Honolulu, Hawaii, USA, 1051-1056. http://dx.doi.org/10.1109/CEC.2002.1004388

Cooper, W. W.; Seiford, L. M.; Tone, K. 2007. Data envelopment analysis: a comprehensive text with models, applications, references and DEA-solver software. New York: Springer.

Deb, K.; Pratap, A.; Agarwal, S.; Meyarivan, T. 2002. A fast and elitist multiobjective genetic algorithm: NSGA-II, IEEE Transactions on Evolutionary Computation 6(2): 182-197.

http://dx.doi.org/10.1109/4235.996017

Eberhart, R. C.; Shi, Y. 2000. Comparing inertia weights and constriction factors in particle swarm optimization, in IEEE Congress on Evolutionary Computation, 16-19 July 2000, San Diego, CA, USA, 84-88. http://dx.doi.org/10.1109/CEC.2000.870279

Falagario, M.; Sciancalepore, F.; Costantino, N.; Pietroforte, R. 2012. Using a DEA-cross efficiency approach in public procurement tenders, European Journal of Operational Research 218(2): 523-529. http://dx.doi.org/10.1016/j.ejor.2011.10.031

Fried, H. O.; Lovell, C. A. K.; Schmidt, S. S. 2008. Efficiency andproductivity, in H. O. Fried, C. A. K. Lovell, S. S. Schmidt (Eds.). The measurement of productive efficiency and productivity growth. Oxford: Oxford University Press.

Gong, M.; Liu, C.; Jiao, L.; Cheng, G. 2010. Hybrid immune algorithm with Lamarckian local search for multi-objective optimization, Memetic Computing 2(1): 47-67.

http://dx.doi.org/ 10.1007/s12293-009-0028-5

Hadi-Vencheh, A. 2011. A new nonlinear model for multiple criteria supplier-selection problem, International Journal of Computer Integrated Manufacturing 24(1): 32-39.

http://dx.doi.org/10.1080/0951192X.2010.527372

Hadi-Vencheh, A.; Niazi-Motlagh, M. 2011. An improved voting analytic hierarchy process-data envelopment analysis methodology for suppliers selection, International Journal of Computer Integrated Manufacturing 24(3): 189-197. http://dx.doi.org/10.1080/0951192X.2011.552528 
Ho, W.; Xu, X.; Dey, P. K. 2010. Multi-criteria decision making approaches for supplier evaluation and selection: a literature review, European Journal of Operational Research 202(1): 16-24. http://dx.doi.org/10.1016/j.ejor.2009.05.009

Kokangul, A.; Susuz, Z. 2009. Integrated analytical hierarch process and mathematical programming to supplier selection problem with quantity discount, Applied Mathematical Modelling 33(3): 1417-1429. http://dx.doi.org/10.1016/j.apm.2008.01.021

Kursawe, F. 1991. A variant of evolution strategies for vector optimization, in Proceedings of the 1st Workshop on Parallel Problem Solving from Nature (PPSN I), 1-3 October 1990, London, UK, 193-197. http://dx.doi.org/10.1007/BFb0029752

Lau, K. H. 2013. Measuring distribution efficiency of a retail network through data envelopment analysis, International Journal of Production Economics 146(2): 598-611.

http://dx.doi.org/10.1016/j.ijpe.2013.08.008

$\mathrm{Li}, \mathrm{X} .2003$. A nondominated sorting particle swarm optimizer for multiobjective optimization, in Proceedings of Genetic and Evolutionary Computation GECCO 2003, 12-16 July 2003, Chicago, IL, USA, 37-48. http://dx.doi.org/10.1007/3-540-45105-6_4

Li, X. B.; Reeves, G. R. 1999. A multiple criteria approach to data envelopment analysis, European Journal of Operational Research 115(3): 507-517.

http://dx.doi.org/10.1016/S0377-2217(98)00130-1

Liao, Z.; Rittscher, J. 2007. A multi-objective supplier selection model under stochastic demand conditions, International Journal of Production Economics 105(1): 150-159.

http://dx.doi.org/10.1016/j.ijpe.2006.03.001

Liu, F. H. F.; Hai, H. L. 2005. The voting analytic hierarchy process method for selecting supplier, International Journal of Production Economics 97(3): 308-317.

http://dx.doi.org/10.1016/j.ijpe.2004.09.005

Liu, Y. 2009. Automatic calibration of a rainfall-runoff model using a fast and elitist multiobjective particle swarm algorithm, Expert Systems with Applications 36(5): 9533-9538.

http://dx.doi.org/10.1016/j.eswa.2008.10.086

Ozkok, B. A.; Tiryaki, F. 2011. A compensatory fuzzy approach to multi-objective linear supplier selection problem with multiple-item, Expert Systems with Applications 38(9): 11363-11368. http://dx.doi.org/10.1016/j.eswa.2011.03.004

Parsopoulos, K. E.; Vrahatis, M. N. 2002. Particle swarm optimization method in multiobjective problems, in Proceedings of the 2002 ACM Symposium on Applied Computing, 11-14 March 2002, Madrid, Spain, 603-607. http://dx.doi.org/10.1145/508791.508907

Rahimi-Vahed, A. R.; Mirghorbani, S. M.; Rabbani, M. 2007. A new particle swarm algorithm for a multi-objective mixed-model assembly line sequencing problem, Soft Computing 11(10): 997-1012. http://dx.doi.org/10.1007/s00500-007-0149-Z

Rodríguez, J. E.; Medaglia, A. L.; Coello Coello, C. A. 2009. Design of a motorcycle frame using neuroacceleration strategies in MOEAs, Journal of Heuristics 15(2): 177-196.

http://dx.doi.org/10.1007/s10732-007-9069-4

Salazar-Lechuga, M.; Rowe, J. E. 2005. Particle swarm optimization and fitness sharing to solve multi-objective optimization problems, in Proceedings of the 2005 IEEE Congress on Evolutionary Computation, 2-5 September 2005, Edinburgh, UK, 1204-1211.

http://dx.doi.org/10.1109/CEC.2005.1554827

Schaffer, J. D. 1985. Multiple objective optimization with vector evaluated genetic algorithms, in Proceedings of the 1st International Conference on Genetic Algorithms, July 1985, Pittsburgh, PA, USA, 93-100.

Sedighizadeh, M.; Faramarzi, H.; Mahmoodi, M. M.; Sarvi, M. 2014. Hybrid approach to FACTS devices allocation using multi-objective function with NSPSO and NSGA-II algorithms in Fuzzy 
framework, International Journal of Electrical Power \& Energy Systems 62: 586-598. http://dx.doi.org/10.1016/j.ijepes.2014.04.058

Srinivas, N.; Deb, K. 1994. Multiobjective optimization using nondominated sorting in genetic algorithms, Evolutionary Computation 2(3): 221-248. http://dx.doi.org/10.1162/evco.1994.2.3.221

Tripathi, P. K.; Bandyopadhyay, S.; Pal, S. K. 2007. Multi-objective particle swarm optimization with time variant inertia and acceleration coefficients, Information Sciences 177(22): 5033-5049. http://dx.doi.org/10.1016/j.ins.2007.06.018

Tsai, S. J.; Sun, T. Y.; Liu, C. C.; Hsieh, S. T.; Wua, W. C.; Chiu, S. Y. 2010. An improved multiobjective particle swarm optimizer for multi-objective problems, Expert Systems with Applications 37(8): 5872-5886. http://dx.doi.org/10.1016/j.eswa.2010.02.018

Ustun, O.; Demirtas, E. A. 2008. An integrated multi-objective decision-making process for multi-period lot-sizing with supplier selection, Omega 36(4): 509-521.

http://dx.doi.org/10.1016/j.omega.2006.12.004

Wang, H. S.; Che, Z. H. 2007. An integrated model for supplier selection decisions in configuration changes, Expert Systems with Applications 32(4): 1132-1140.

http://dx.doi.org/10.1016/j.eswa.2006.02.015

Wang, H. S.; Che, Z. H. 2008. A multi-phase model for product part change problems, International Journal of Production Research 46(10): 2797-2825.

http://dx.doi.org/10.1080/00207540600999144

Wang, Z.; Yang, Z.; Tang, K.; Yao. X. 2009. Adaptive differential evolution for multi-objective optimization, in Proceeding of the 20th International Conference on Multiple Criteria Decision Making, 21-26 June 2009, Chengdu, Jiuzhaigou, China, 9-16.

http://dx.doi.org/10.1007/978-3-642-02298-2_2

Weber, C. A.; Current, J. R.; Benton, W. C. 1991. Vendor selection criteria and methods, European Journal of Operational Research 50(1): 2-18. http://dx.doi.org/10.1016/0377-2217(91)90033-R

Zhang, L. P.; Yu, H. J.; Hu, S. X. 2005. Optimal choice of parameters for particle swarm optimization, Journal of Zhejiang University Science A 6(6): 528-534.

http://dx.doi.org/10.1007/BF02841760

Zitzler, E.; Deb, K.; Thiele, L. 2000. Comparison of multiobjective evolutionary algorithms: empirical results, Evolutionary Computation 8(2): 173-195. http://dx.doi.org/10.1162/106365600568202

Z. H. CHE is a Professor in Department of Industrial Engineering and Management at National Taipei University of Technology in Taiwan. He received his PhD. in Industrial Engineering and Management at National Chiao Tung University in Taiwan. His current research interests include production/ operations management, supply chain management, information management, and applications of meta-algorithms.

Y. F. CHANG received his Master in Industrial Engineering and Management at National Taipei University of Technology in Taiwan. His research interests include supply chain management and applications of meta-algorithms. 